University of Nebraska - Lincoln

DigitalCommons@University of Nebraska - Lincoln

2011

Numerical simulations of single and multiple scattering by fractal ice clusters

Janna M. Dlugach

Main Astronomical Observatory of the National Academy of Sciences of Ukraine

Michael I. Mishchenko

NASA Goddard Institute for Space Studies, New York

Daniel W. Mackowski

Auburn University Main Campus

Follow this and additional works at: https://digitalcommons.unl.edu/nasapub

Part of the Physical Sciences and Mathematics Commons

Dlugach, Janna M.; Mishchenko, Michael I.; and Mackowski, Daniel W., "Numerical simulations of single and multiple scattering by fractal ice clusters" (2011). NASA Publications. 51.

https://digitalcommons.unl.edu/nasapub/51

This Article is brought to you for free and open access by the National Aeronautics and Space Administration at DigitalCommons@University of Nebraska - Lincoln. It has been accepted for inclusion in NASA Publications by an authorized administrator of DigitalCommons@University of Nebraska - Lincoln. 


\title{
Numerical simulations of single and multiple scattering by fractal ice clusters
}

\author{
Janna M. Dlugach ${ }^{a}$, Michael I. Mishchenko ${ }^{b, *}$, Daniel W. Mackowski ${ }^{c}$ \\ a Main Astronomical Observatory of the National Academy of Sciences of Ukraine, 27 Zabolotny Street, 03680 Kyiv, Ukraine \\ ${ }^{\mathrm{b}}$ NASA Goddard Institute for Space Studies, 2880 Broadway, New York, NY 10025, USA \\ ${ }^{\mathrm{c}}$ Department of Mechanical Engineering, Auburn University, AL 36849, USA
}

\section{A R T I C L E I N F O}

Available online 5 February 2011

Keywords:

Electromagnetic scattering

Radiative transfer

Coherent backscattering

Fractal cluster

Circular polarization ratio

\begin{abstract}
A B S T R A C T
We consider the scattering model in the form of a vertically and horizontally homogeneous particulate slab of an arbitrary optical thickness composed of widely separated fractal aggregates built of small spherical ice monomers. The aggregates are generated by applying three different approaches, including simulated cluster-cluster aggregation (CCA) and diffusion-limited aggregation (DLA) procedures. Having in mind radar remote-sensing applications, we report and analyze the results of computations of the backscattering circular polarization ratio obtained using efficient superposition $T$-matrix and vector radiative-transfer codes. The computations have been performed at a wavelength of $12.6 \mathrm{~cm}$ for fractal aggregates with the following characteristics: monomer refractive index $m=1.78+\mathrm{i} 0.003$, monomer radius $r=1 \mathrm{~cm}$, monomer packing density $p=0.2$, overall aggregate radii $R$ in the range $4 \leq R \leq 10 \mathrm{~cm}$ and fractal dimensions $D_{f}=2.5$ and 3 .

We show that for aggregates generated with simulated CCA and DLA procedures, the respective values of the backscattering circular polarization ratio differ weakly for $D_{f}=2.5$, but the differences can increase somewhat for $D_{f}=3$, especially in case of an optically semi-infinite medium. For aggregates with a spheroidal overall shape, the dependence of the circular polarization ratio on the cluster morphology can be quite significant and increases with increasing the aspect ratio of the circumscribing spheroid.
\end{abstract}

Published by Elsevier Ltd.

\section{Introduction}

Scattering of electromagnetic waves by a particulate medium composed of clusters of ice monomers is a subject of potential importance to the discipline of remote sensing of the Earth and other solar system objects. For example, the knowledge of the scattering properties of such a medium is needed for the interpretation of monostatic radar observations of terrestrial snow and ice surfaces [1], the Galilean satellites of Jupiter [2], and

\footnotetext{
* Corresponding author. Fax: +1 2126785622 .

E-mail address: mmishchenko@giss.nasa.gov (M.I. Mishchenko).
}

Saturn's rings [3]. The conventional approach to solving this problem involves the calculation of single-scattering characteristics of an ice cluster with one of the numerical techniques developed over the past decades [4-9] followed by a vector radiative-transfer/coherent-backscattering computation [10-14]. It is clear that when performing such computations, a specific theoretical model of the aggregate structure must be adopted, and it should be expected that scattering characteristics of clusters can be affected in some way by their morphology. A relevant question is how strong these effects can be.

In this paper, we consider a simple model of a particulate scattering medium in the form of a slab composed of fractal aggregates built of small spherical ice monomers. 
Having in mind radar remote-sensing applications, we examine potential effects of the cluster morphology on the behavior of the backscattering circular polarization ratio $\mu_{\mathrm{c}}$. This quantity is typically measured with monostatic polarimetric radars and has been demonstrated to be highly indicative of microphysical characteristics of the scattering medium (e.g., [12,15] and references therein). Another motivation for selecting this quantity for a sensitivity analysis is the issue of measurement accuracywhile absolute measurements of radar cross sections may suffer from several sources of errors and uncertainties, most systematic uncertainties cancel out in the computation of the circular polarization ratio $\mu_{\mathrm{C}}$, thereby resulting in a more reliably determined quantity (see, e.g. [3]).

\section{Ice cluster models and computational techniques}

Let the scattering medium be a plane-parallel slab composed of randomly and sparsely distributed aggregates built of spherical ice monomers. The slab is illuminated by a parallel beam of light, with pairs $\left(\theta_{0}, \varphi_{0}\right)$ and $(\theta, \varphi)$ of zenith (polar) and azimuth angles specifying the directions of incidence and reflection, respectively [12]. As usual, the zenith angles are measured with respect to the outward normal to the upper boundary of the slab.

The first step in the computation of single-scattering characteristics of an ice aggregate is to generate random monomer positions. As an initial model of ice aggregates we adopt a fractal cluster that can be described by the following statistical scaling law [16]:

$N_{s}=k_{0}\left(\frac{R_{g}}{r}\right)^{D_{f}}$,

where $r$ is the monomer radius, $1 \leq D_{\mathrm{f}} \leq 3$ is the fractal dimension, $k_{0}$ is the pre-factor, $N_{s}$ is the number of spherical monomers in the cluster, and $R_{\mathrm{g}}$, called the radius of gyration, is a measure of the overall cluster radius. The parameters $N_{s}, D_{f}$, and $k_{0}$ specify the morphology of a fractal aggregate.

In this study, we apply the following two algorithms to generate monomer positions in a fractal cluster.

1. The simulated cluster-cluster aggregation (CCA) procedure [17]. This is a sequential algorithm for building pseudo-random monomer coordinates for a fractal aggregate. At any point in the sequence, the method generates a set of $N=N_{1}+N_{2}$ monomer coordinates by the aggregation of sets containing $N_{1}$ and $N_{2}$ coordinates, subject to the constraints that the positions of the new set satisfy Eq. (1) identically for given $k_{0}$ and $D_{f}$ and that each monomer touches at least one other monomer.

2. The simulated diffusion-limited aggregation (DLA) method [18] in which the generation procedure starts with a pair of spheres in contact for pre-set $k_{0}$ and $D_{f}$ values and adds a single monomer at a time. Compared with the CCA procedure, the DLA algorithm is less realistic, but it can be used for a wider range of values of the fractal parameters.

We also use an alternative approach capable of generating clusters with spheroidal as well as nearly spherical overall shapes. Specifically, this procedure generates clusters composed of spherical monomers that lie randomly within the surface of a spheroid, subject to the constraints that no two spheres overlap and that each sphere is in contact with at least one neighbor. Note that such clusters are not described by Eq. (1). The respective monomer packing density $p$ is defined by

$p=\frac{N_{s} r^{3}}{E R_{a}^{3}}$,

where $R_{a}$ is the axial radius of the spheroid and $E$ is the axial ratio such that $E>1$ represents a prolate spheroid.

Note that the overall shape of fractals generated by using both the CCA and DLA algorithms for fractal dimension values close to 3 is nearly spherical. Therefore, Eq. (2) with $E=1$ also applies to such CCA- and DLAgenerated clusters.

Fig. 1 shows examples of aggregates generated by using the CCA procedure and Eq. (2). The overall radius $R$ (i.e., the radius of the smallest circumscribing sphere) and packing density $p$ are equal to $8 \mathrm{~cm}$ and 0.2 , respectively.

In recent years, the numerically exact superposition T-matrix method [19] has been applied extensively to the computation of single scattering by a multi-sphere cluster in random orientation (see, e.g., [20] and references therein). Therefore, we compute the elements of the single-scattering Stokes matrix for the generated clusters using the corresponding FORTRAN program publicly available on-line [21]. Then the resulting ensemble-averaged single-scattering characteristics of fractal clusters are used to compute the elements of the diffuse Stokes a

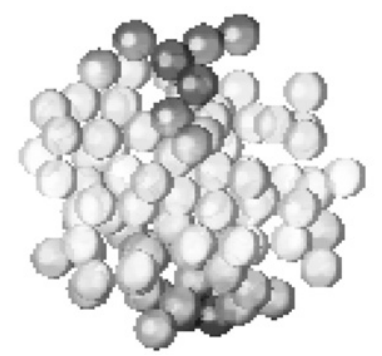

b

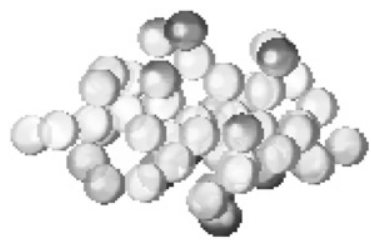

C

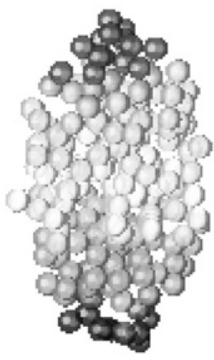

Fig. 1. Aggregate particles with (a) nearly spherical, (b) oblate, and (c) prolate shapes. 
reflection matrix

$\mathbf{R}=\left[\begin{array}{cccc}R_{11} & R_{12} & 0 & 0 \\ R_{12} & R_{22} & 0 & 0 \\ 0 & 0 & R_{33} & R_{34} \\ 0 & 0 & -R_{34} & R_{44}\end{array}\right]$

corresponding to the case of an exact backscattering direction (i.e., $\theta=\pi-\theta_{0}$ and $\varphi=\varphi_{0}+\pi$ ). For this purpose we employ a vector radiative-transfer code based on the numerical solution of Ambarzumian's nonlinear integral equation $[22,23]$ in the case of a semi-infinite homogeneous slab, whereas for a finite slab we use a numerically exact computer code based on the invariant imbedding technique [24]. Then the backscattering circular polarization ratio is computed according to the formula (see, e.g., [12])

$\mu_{\mathrm{C}}=\frac{R_{11}^{1}+R_{44}^{1}+2 R_{11}^{\mathrm{M}}+2 R_{44}^{\mathrm{M}}}{R_{11}^{1}-R_{44}^{1}+R_{11}^{\mathrm{M}}+R_{22}^{\mathrm{M}}-R_{33}^{\mathrm{M}}-R_{44}^{\mathrm{M}}}$,

where the superscript " 1 " denotes the contribution of the first-order-scattering and " $\mathrm{M}$ " denotes the diffuse multiple-scattering contribution. Note that Eq. (4) incorporates fully the effect of coherent backscattering.

\section{Numerical results}

In this section we present the results of computations performed for a plane-parallel homogeneous slab composed of fractal ice aggregates. The refractive index $m=m_{\mathrm{R}}+\mathrm{i} m_{\mathrm{I}}$ (with $\mathrm{i}$ being the square root of minus one) is adopted to be equal to $1.78+\mathrm{i} 0.003$, which corresponds to weakly contaminated water ice at a wavelength of $12.6 \mathrm{~cm}$ [25]. Note that this wavelength was used extensively in radar polarimetric measurements of Saturn's rings $[3,26]$. The monomer packing density $p$ is fixed at 0.2 , the monomer radius is $r=1 \mathrm{~cm}$, and the overall cluster radius $R$ is varied from 4 up to $10 \mathrm{~cm}$. As stated above, we consider three types of aggregates-clusters of nearly spherical overall shape described by Eq. (1) and generated by using the CCA and DLA procedures as well as clusters of spheroidal (including spherical) overall shape described by Eq. (2). Below we depict and discuss the results obtained.

\subsection{Aggregate particles of nearly spherical overall shape}

In this case, the number of monomers $N_{s}$ and the fractal pre-factor $k_{0}$ can be found from the relations

$N_{s}=\frac{p R^{3}}{r^{3}}$,

$N_{s}=k_{0}\left(\frac{R-r}{r}\right)^{D_{f}}$

In Table 1 we list the values of $N_{\mathrm{s}}$ and $k_{0}$ derived from Eqs. (5) and (6) for several values of the overall fractal radius in the range $4 \mathrm{~cm} \leq R \leq 10 \mathrm{~cm}$ and $D_{f}=2.5$ and 3 . In order to obtain the requisite single-scattering characteristics of fractals for each value of the overall radius $R$ and the specified fractal parameters, we generated an ensemble of ten fractal-parameter-equivalent realizations of an aggregate, computed the orientation-averaged singlescattering characteristics for each realization using the superposition $T$-matrix method, and then averaged the results over the ensemble. The necessity of performing such ensemble averaging was discussed in [27]. A similar ensemble-averaging procedure was applied to nearly spherical clusters generated using Eq. (2).

Table 1

Fractal parameters used to model nearly spherical clusters.

\begin{tabular}{lcccr}
\hline$R(\mathrm{~cm})$ & 4 & 6 & 8 & 10 \\
$N_{\mathrm{s}}$ & 13 & 43 & 102 & 200 \\
$k_{0}\left(D_{\mathrm{f}}=2.5\right)$ & 0.83 & 0.77 & 0.79 & 0.82 \\
$k_{0}\left(D_{\mathrm{f}}=3\right)$ & 0.48 & 0.34 & 0.30 & 0.27 \\
\hline
\end{tabular}
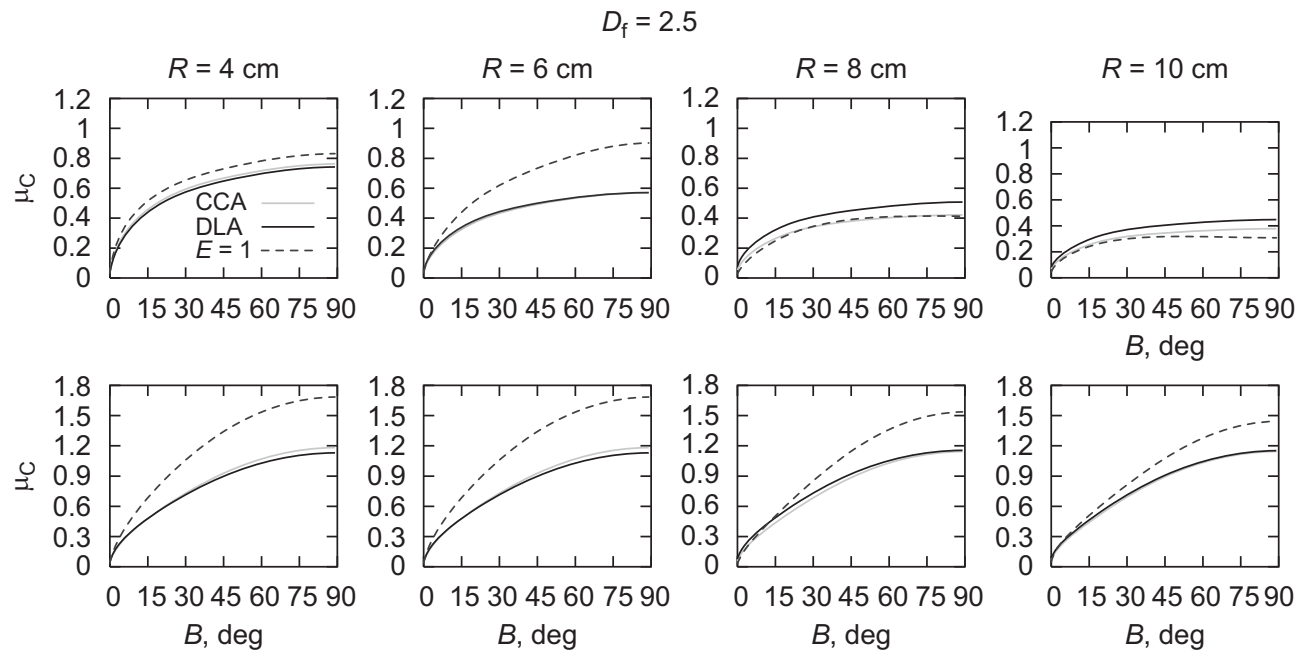

$B$, deg

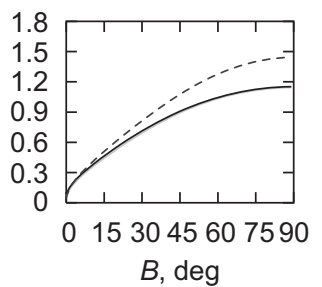

Fig. 2. Angular dependence of backscattering circular polarization ratio $\mu_{\mathrm{C}}$ for a plane-parallel slab composed of ice clusters with the fractal dimension $D_{\mathrm{f}}=2.5$. The slab optical thickness is 2 (top row) and $\infty$ (bottom row). 

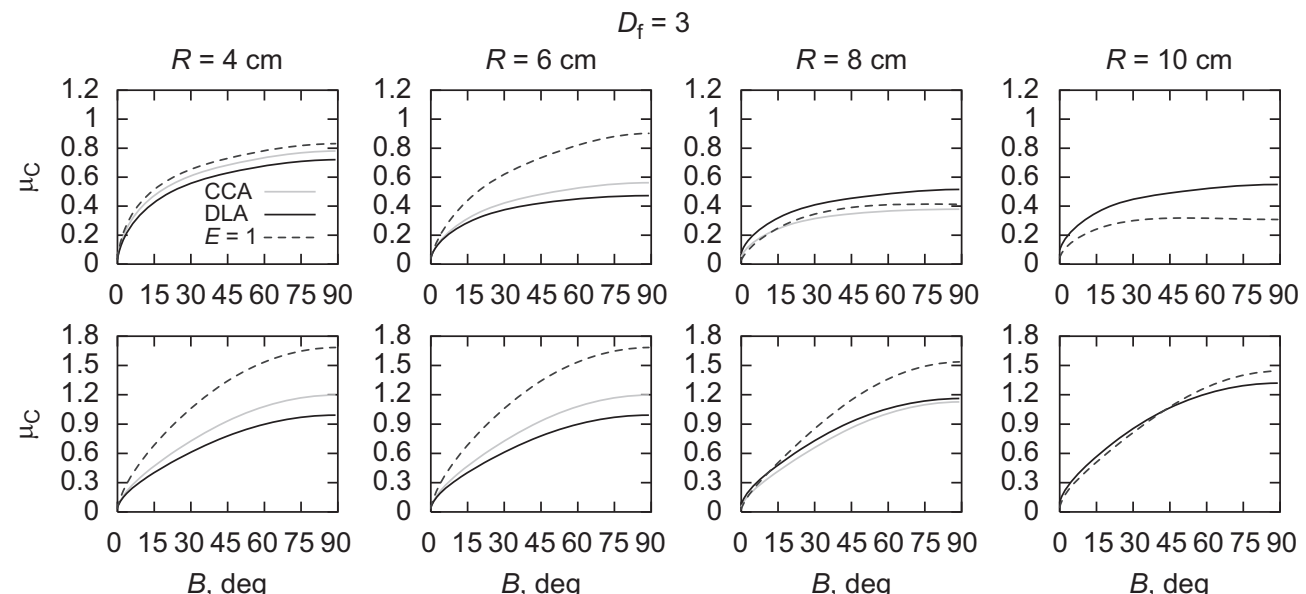

Fig. 3. As in Fig. 2, but for the fractal dimension $D_{f}=3$.
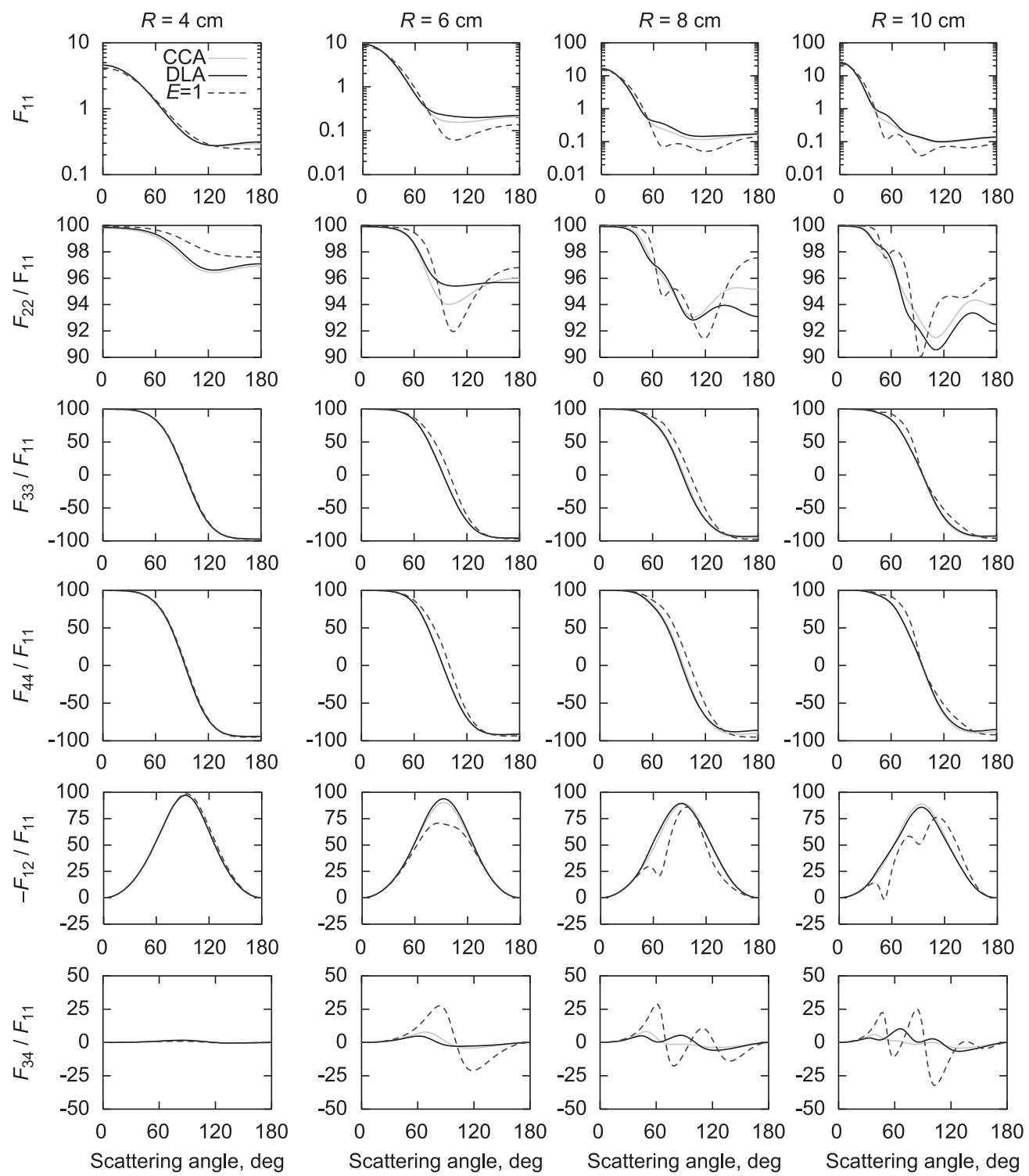

Fig. 4. Ensemble-averaged single-scattering matrix elements versus scattering angle for $D_{f}=2.5$. 
Figs. 2 and 3 depict the computed dependences of the backscattering circular polarization ratio $\mu_{\mathrm{C}}$ on the so-called tilt angle $B=\pi / 2-\theta$. Here, we present the results of computations performed for slabs with two values of optical thickness: $\tau=2$ and $\infty$. The slabs are composed of fractals with $D_{\mathrm{f}}=2.5$ (Fig. 2) and 3 (Fig. 3) generated by employing the CCA and DLA procedures as well as by using Eq. (2) with $E=1$ (the $E=1$ curves are the same in Figs. 2 and 3). It should be noted that for the fractal parameters adopted, the CCA procedure fails to generate aggregates with $D_{f}=3$ and $R>8 \mathrm{~cm}$.

We see a significant angular dependence of the polarization ratio, which becomes more pronounced with increasing $\tau$ but weakens with increase in the value of the overall radius $R$ for $\tau=2$. For $D_{\mathrm{f}}=2.5$ and $\tau=2$, one can see that for the clusters generated with the CCA and DLA algorithms, the values of $\mu_{\mathrm{C}}$ are very close, weak deviations occurring only for $R=10 \mathrm{~cm}$ and $B>60^{\circ}$. In the case
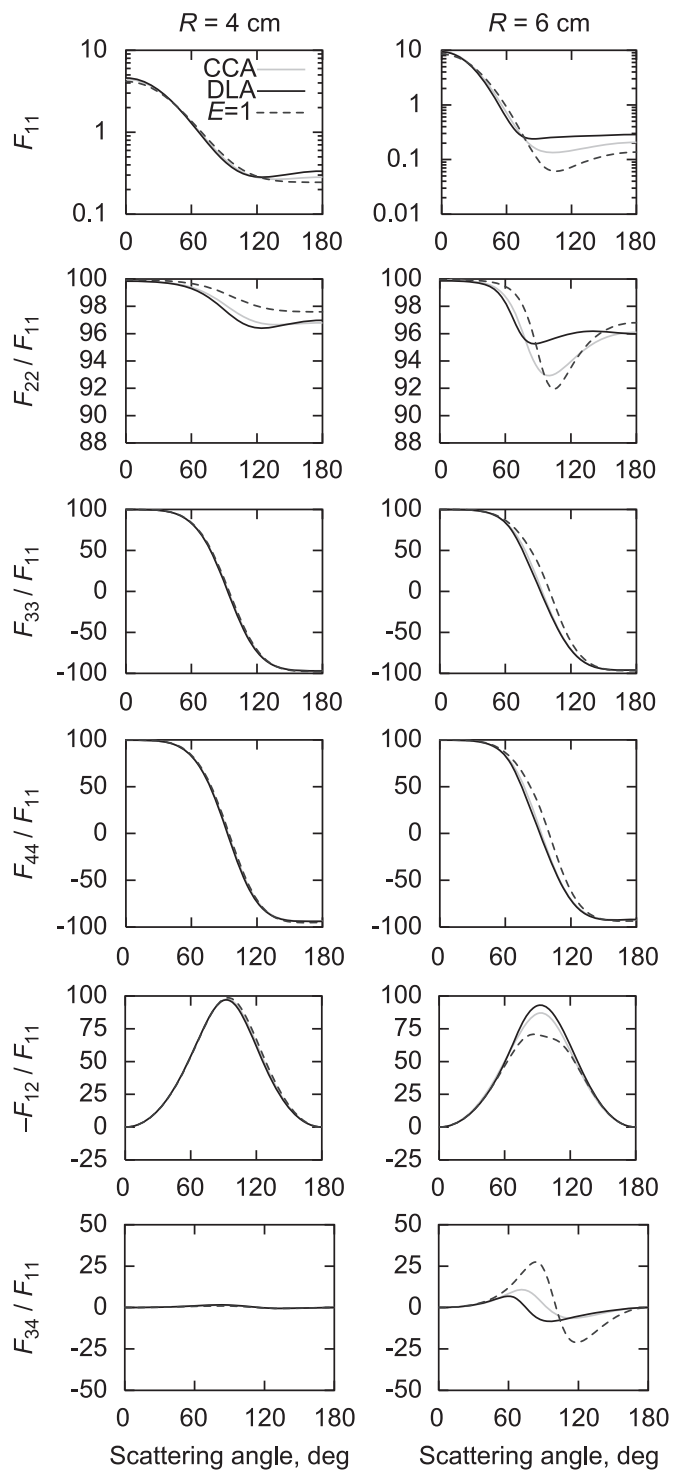

of a semi-infinite medium, the respective $\mu_{\mathrm{C}}$ curves almost overlap. The results depicted in Figs. 2 and 3 show that the differences in the values of $\mu_{\mathrm{C}}$ due to the application of different cluster-generating procedures increase somewhat with $D_{\mathrm{f}}$, especially in the case of a semi-infinite medium.

As regards the case of clusters described by Eq. (2), significant differences in the values of $\mu_{\mathrm{C}}$ can be observed relative to the respective CCA and DLA values. Note that when $\tau=2$, maximal differences occur for the overall fractal radius $R=6 \mathrm{~cm}$, while for a semi-infinite layer, the respective $\mu_{\mathrm{C}}$ values differ significantly for almost all values of $R$ considered. Furthermore, it is seen that for $\tau=\infty$, the dependence of $\mu_{C}$ on the type of clustergeneration procedure is much stronger (except in the case of $R=10 \mathrm{~cm}$ ).

In order to give a plausible explanation for these differences in the computed $\mu_{\mathrm{C}}$-values, we depict in Figs. 4 and 5
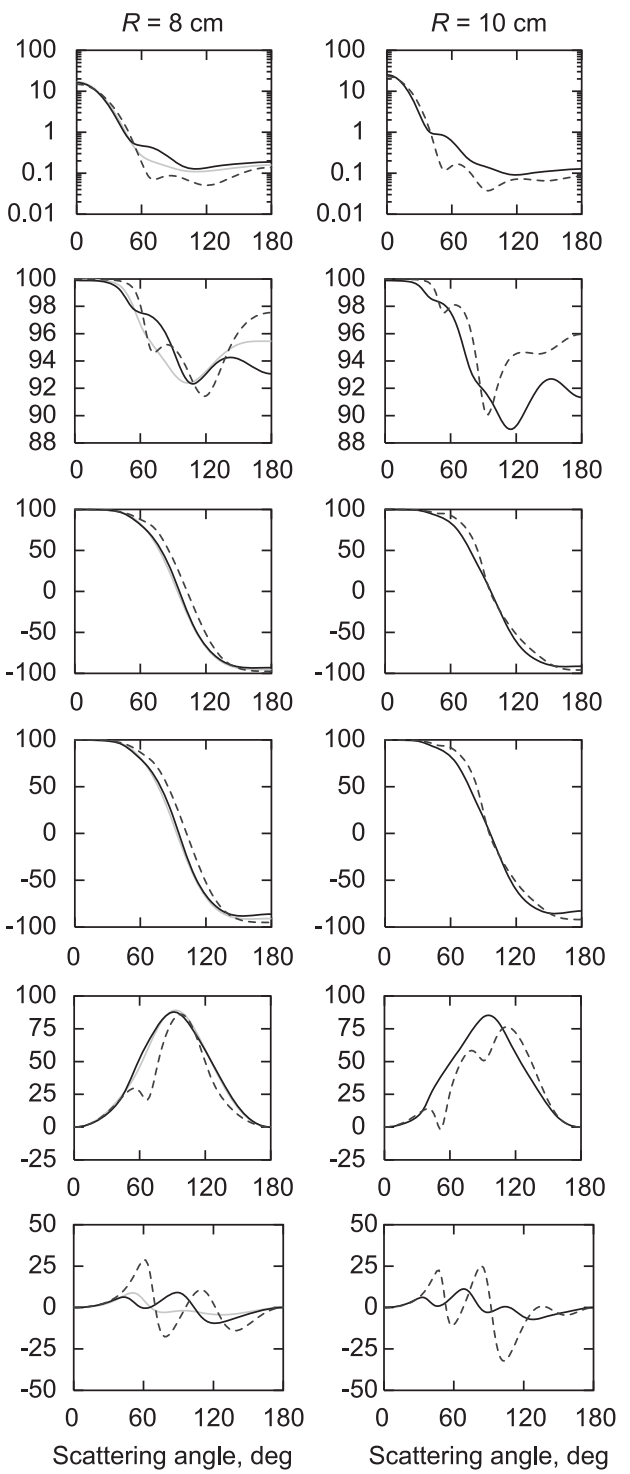

Fig. 5. As in Fig. 4, but for $D_{\mathrm{f}}=3$. 
the elements of the ensemble-averaged normalized singlescattering Stokes matrix $\mathbf{F}$ [5] as functions of the scattering angle (the $E=1$ curves are the same in Figs. 4 and 5). According to Section 14.3 of [12], the elements of the firstorder-scattering reflection matrix entering Eq. (4) are proportional to the respective elements of $\mathbf{F}$ :

$\mathbf{R}^{1}=\frac{\varpi[1-\exp (-2 \tau / \cos \theta)]}{8 \cos \theta} \mathbf{F}\left(180^{\circ}\right)$,

$\left.\mathbf{R}^{1}\right|_{\tau \rightarrow \infty}=\frac{\varpi}{8 \cos \theta} \mathbf{F}\left(180^{\circ}\right)$,

where $\varpi$ is the single-scattering albedo. One can see that among the three cluster-generating procedures used, the differences in the corresponding backscattering values of the diagonal elements $F_{22}, F_{33}$, and $F_{44}$ entering Eq. (4) are very small, while $F_{44}$ is very close to $-F_{11}$. This is consistent with the fact that in the limit $\theta \rightarrow 90^{\circ}$ (or $B \rightarrow 0^{\circ}$ i.e., when the first-order-scattering contribution to the reflection matrix dominates) the differences between all three circular polarization ratios and the ratios themselves essentially vanish. We can thus conclude that the main contribution to the differences in the respective circular polarization ratios is caused by multiple scattering.

\subsection{Aggregate particles of spheroidal overall shape}

In Table 2 we list the values of $N_{s}$ derived from Eq. (2) for the packing density $p=0.2$ and various values of the overall radius of spheroids $R$ and the axial ratio $E$ (we remind that values $E>1$ correspond to prolate spheroids). Note that for the same values of $R$ the number of spherical monomers forming the cluster differs substantially for oblate and prolate spheroids having the same aspect ratio. Therefore, it is natural to expect that the corresponding values of the circular polarization ratio will also differ noticeably.

Figs. 6 and 7 visualize the results of computations of the backscattering circular polarization ratio $\mu_{\mathrm{C}}$ obtained for various values of the aggregate overall radius $R$, optical thickness values $\tau=2$ and $\infty$, and axial ratios $E=1.2$ and $1 / 1.2$ (Fig. 6) as well as $E=2$ and $1 / 2$ (Fig. 7). Again,
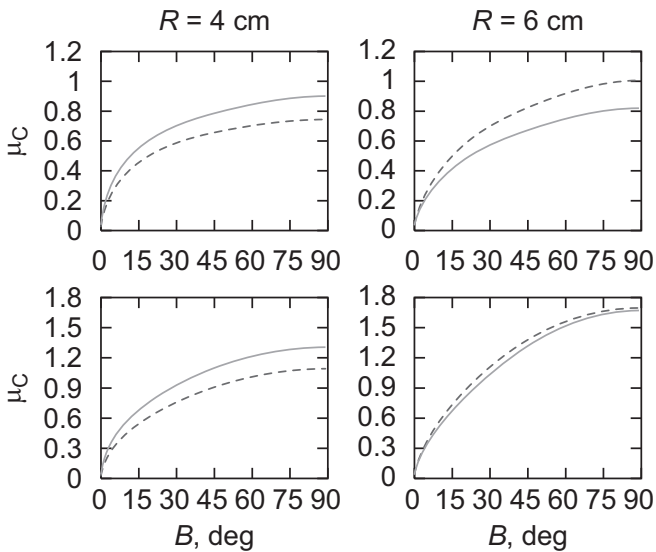

in these computations we used the same ensemble averaging of the single-scattering characteristics as for the nearly spherical clusters. One can see that with a few exceptions, the values of $\mu_{C}$ differ substantially for the slabs composed of fractals of overall oblate and prolate spheroidal shapes with the same aspect ratio. As could be expected, the magnitude of these differences grows with increasing asphericity of the overall cluster shape. However, the character of this difference is not monotonous and depends on the value of $R$, especially for $E=2$ and $1 / 2$. Specifically, $\mu_{\mathrm{C}}(E=1 / 1.2)<\mu_{\mathrm{C}}(E=1.2)$ and $\mu_{\mathrm{C}}(E=1 / 2)<$ $\mu_{\mathrm{C}}(E=2)$ for smaller $R$, while $\mu_{\mathrm{C}}(E=1 / 1.2)>\mu_{\mathrm{C}}(E=1.2)$ and $\mu_{\mathrm{C}}(E=1 / 2)>\mu_{\mathrm{C}}(E=2)$ for larger $R$. This suggests strongly that the differences in the respective $N_{s}$ values (Table 2) are not the main cause of the observed differences in the backscattering circular polarization ratios.

\section{Discussion and conclusion}

The results of our computations performed with the superposition $T$-matrix method and numerically exact vector radiative-transfer codes demonstrate that for media composed of aggregates generated by the simulated CCA and DLA procedures, the respective values of the backscattering circular polarization ratio differ from each other rather weakly in the case of the fractal dimension $D_{\mathrm{f}}=2.5$. For $D_{f}=3$, this difference can increase somewhat, especially for a semi-infinite medium. If, however, nearly spherical aggregates are generated using Eq. (2) then the corresponding $\mu_{C}$ values can differ quite substantially from the respective CCA and DLA values.

Table 2

Number of monomers $N_{\mathrm{s}}$ in spheroidal clusters.

\begin{tabular}{lrrrr}
\hline$R(\mathrm{~cm})$ & \multicolumn{1}{l}{$E$} & & \\
\cline { 2 - 5 } & 1.2 & 2 & $1 / 1.2$ & $1 / 2$ \\
\hline 4 & 16 & 26 & 11 & 7 \\
6 & 52 & 87 & 36 & 22 \\
8 & 123 & 205 & 86 & 51 \\
10 & 240 & 400 & 167 & 100 \\
\hline
\end{tabular}
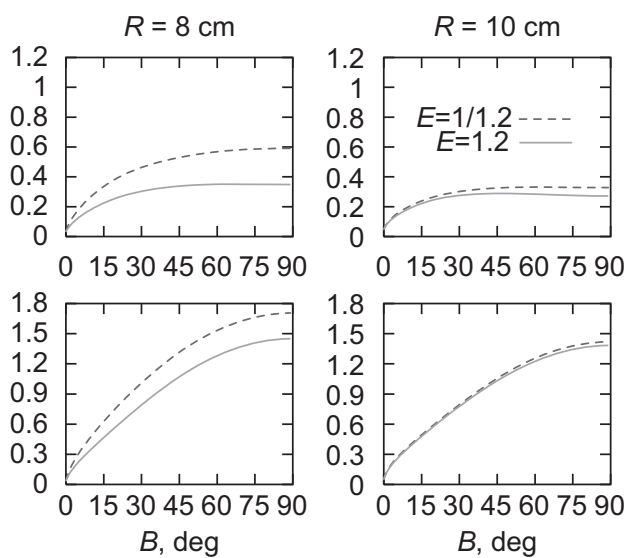

Fig. 6. Angular dependence of backscattering circular polarization ratio $\mu_{\mathrm{C}}$ for a plane-parallel slab composed of ice clusters with oblate $(E=1 / 1.2)$ and prolate $(E=1.2)$ spheroidal overall shapes. The slab optical thickness is 2 (top row) and $\infty$ (bottom row). 

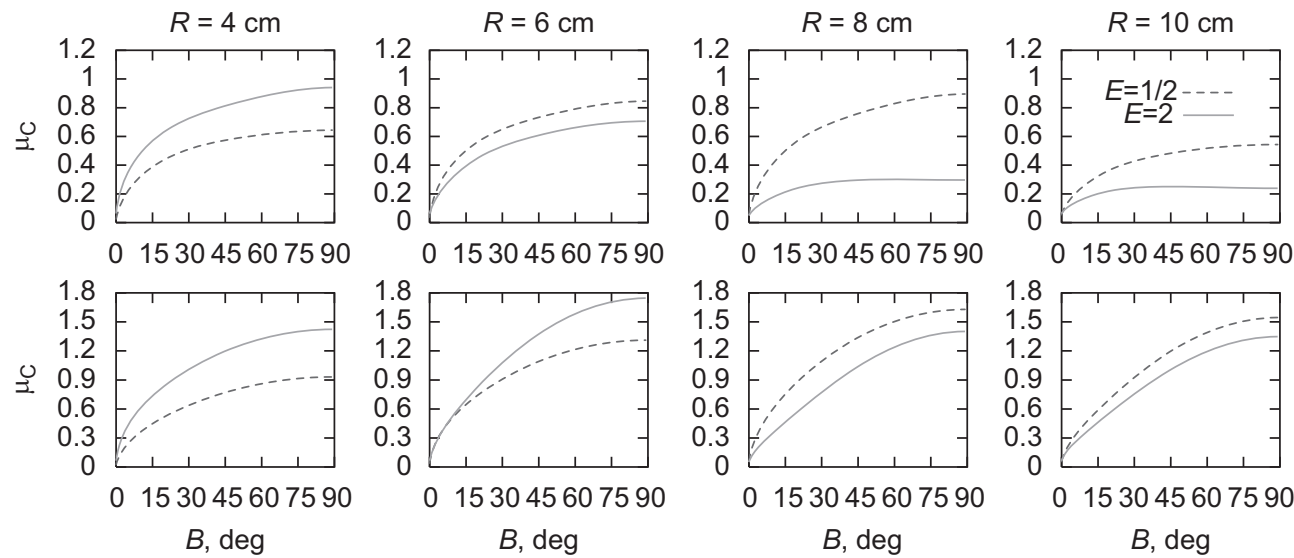

Fig. 7. As in Fig. 6, but for spheroidal aggregates with $E=1 / 2$ and 2 .

Interestingly, the phase-function differences in the upper rows of Figs. 4 and 5 can exceed a factor of 3 at some scattering angles. Since in all cases the same numbers of spherical monomers were packed into identical spherical volumes, these phase-function differences illustrate once again the potentially strong residual effect of a specific aggregation process on light scattering.

Eq. (2) can also be used to generate clusters with spheroidal overall shapes. Then the resulting dependence of $\mu_{\mathrm{C}}$ on the aggregate morphology can be quite significant and complex and increases with increase in the degree of asphericity of the overall aggregate shape.

Taking into account that our numerical simulations were performed for clusters composed of ice monomers at a wavelength of $12.6 \mathrm{~cm}$, we expect that the above results and conclusions can be useful in future analyses of radar polarimetric observations of ice-covered surfaces of solar system bodies (such as Saturn's rings, the Galilean satellites of Jupiter, etc.).

\section{Acknowledgment}

This research was supported by the NASA Radiation Sciences Program managed by Hal Maring.

\section{References}

[1] Rignot E. Backscatter model for the unusual radar properties of the Greenland ice sheet. J Geophys Res 1995;100:9389-400.

[2] Ostro SJ. Planetary radar astronomy. Rev Mod Phys 1993;65: 1235-79.

[3] Nicholson PD, French RG, Campbell DB, et al. Radar imaging of Saturn's rings. Icarus 2005;177:32-62.

[4] Mishchenko MI, Hovenier JW, Travis LD, editors. San Diego: Academic Press; 2000.

[5] Mishchenko MI, Travis LD, Lacis AA Scattering, absorption, and emission of light by small particles. Cambridge, UK: Cambridge University Press; 2002 (〈http://www.giss.nasa.gov/staff/mmish chenko/books.html)>

[6] Kahnert FM. Numerical methods in electromagnetic scattering theory. J Quant Spectrosc Radiat Transfer 2003;79/80:775-824.

[7] Doicu A, Wriedt T, YuA Eremin. Light scattering by systems of particles. Null-field method with discrete sources: theory and programs. Berlin: Springer; 2006.
[8] Borghese F, Denti P, Saija R. Scattering from model nonspherical particles. Theory and applications to environmental physics. Berlin: Springer; 2007.

[9] Wriedt T. Light scattering theories and computer codes. J Quant Spectrosc Radiat Transfer 2009;110:833-43.

[10] Tsang L, Kong JA, Shin RT. Theory of microwave remote sensing. New York: Wiley; 1985.

[11] Hovenier JW, Van der Mee C, Domke H. Transfer of polarized light in planetary atmospheres-basic concepts and practical methods. Dordrecht, The Netherlands: Kluwer; 2004.

[12] Mishchenko MI, Travis LD, Lacis AA. Multiple scattering of light by particles: radiative transfer and coherent backscattering. Cambridge, UK: Cambridge University Press; 2006.

[13] Mishchenko MI, Dlugach JM. Weak localization of electromagnetic waves and radar polarimetry of Saturn's rings. Mon Not R Astron Soc 2008;389:1665-74.

[14] Mishchenko MI, Rosenbush VK, Kiselev NN, Lupishko DF, Tishkovets VP, Kaydash VG, et al. Polarimetric remote sensing of solar system objects. Kyiv: Akademperiodyka 2010. (arXiv:1010.1171).

[15] Stephens GL. Remote sensing of the lower atmosphere. New York: Oxford University Press; 1994.

[16] Sorensen CM. Light scattering by fractal aggregates: a review. Aerosol Sci Technol 2001;35:648-87.

[17] Mackowski DW. A simplified model to predict the effects of aggregation on the absorption properties of soot particles. J Quant Spectrosc Radiat Transfer 2006;100:237-49.

[18] Mackowski DW. Electrostatistics analysis of radiative absorption by sphere clusters in the Rayleigh limit: application to soot particles. Appl Opt 1995:34:3535-45.

[19] Mackowski DW, Mishchenko MI. Calculation of the T-matrix and the scattering matrix for ensembles of spheres. J Opt Soc Am A 1996:13:2266-78.

[20] Mishchenko MI, Videen G, Babenko VA, et al. Comprehensive T-matrix reference database: a 2007-2009 update. J Quant Spectrosc Radiat Transfer 2010;111:650-8.

[21] Mackowski DW ftp://ftp.eng.auburn.edu/pub/dmckwski/scatcodes/ index.html.

[22] de Rooij WA. Reflection and transmission of polarized light by planetary atmospheres. PhD dissertation. Amsterdam: Vrije Universiteit; 1985.

[23] Mishchenko MI. Diffuse and coherent backscattering by discrete random media. I. Radar reflectivity, polarization ratios, and enhancement factors for a half-space of polydisperse, nonabsorbing and absorbing spherical particles. J Quant Spectrosc Radiat Transfer 1996;56:673-702.

[24] Mishchenko MI. The fast invariant imbedding method for polarized light: computational aspects and numerical results for Rayleigh scattering. J Quant Spectrosc Radiat Transfer 1990;43:163-71.

[25] Warren SG, Brandt RE. Optical constants of ice from the ultraviolet to the microwave: a revised compilation. J Geophys Res 2008;113: D14220.

[26] Ostro SJ, Pettengill GH, Campbell DB. Radar observations of Saturn's rings at intermediate tilt angles. Icarus 1980;41:381-8.

[27] Mishchenko MI, Dlugach JM. Radar polarimetry of Saturn's rings: modeling ring particles as fractal aggregates built of small ice monomers. J Quant Spectrosc Radiat Transfer 2009;110:1706-12. 